\title{
Dilation as a precursor in a continuous granular fault
}

\author{
Victor Levy dit Vehel ${ }^{1, *}$, Takahiro Hatano ${ }^{2}$, Loïc Vanel $^{2}$, Knut Jørgen Måløy ${ }^{3}$, and Osvanny Ramos ${ }^{1, * *}$ \\ ${ }^{1}$ Institut Lumière Matière, UMR5306 Université Lyon 1-CNRS, Université de Lyon 69622 Villeurbanne, France \\ ${ }^{2}$ Department of Earth and Space Science, Osaka University, 560-0043 Osaka, Japan \\ ${ }^{3}$ PoreLab, The Njord Centre, Department of Physics, University of Oslo, P. O. Box 1048, 0316 Oslo, Norway
}

\begin{abstract}
We analyze the dilation of the system in a cylindrical granular fault consisting of one single layer of disks submitted to both normal pressure and continuous and slow shear, which results in intermittent and sudden energy release events that reproduce the main laws of seismicity. The dilation of the system can be separated into two parts: a smooth increase of dilation, plus sudden changes both contracting and dilating the medium, which are correlated to abrupt jumps -both positive and negative- in the measured resisting torque. We explain the four possible (and existing) general scenarios combining those two variables: dilation jumps and torque jumps, thanks to the assumption of an optimal local angle in the direction of force chains, and each reorganization of the structure as a replacement of the force chain holding most of the applied stress. The average rate of increase of global dilation varies monotonically with the size of the energy release event, making dilation a plausible candidate to predict catastrophic events in such earthquake-like systems.
\end{abstract}

\section{Introduction}

Granular systems are often used as analogue experiments mimicking the behavior of a tectonic fault [1-8]. The slow accumulation of energy that is released by sudden events, illustrated with a sandpile in the late 80s [9], and the intrinsic disorder of a granular pile, may indeed generate an intermittent dynamics with a power law distribution of event sizes that qualitatively resembles the one of real earthquakes [3]. The existence of granular gauges as a result of the fracturing processes in the boundaries of adjacent tectonic plates moving relative to each other [10], validates the use of a granular fault as a plausible analogue to a tectonic one. How far the analogies between both systems can hold? Recently an original experimental system developed in our lab in Lyon was able to quantitatively and simultaneously reproduce, with a statistics of more than 1.8 millions of acoustic events, three main laws of seismicity [7]. This strongly suggests that both systems share a common physics and calls for a better understanding of this complex behavior at the granular level. Dilation induced by shear is a classical [11] an intuitive phenomenon that we will analyze under our earthquake-like dynamics.

Preliminary results [12] from our experimental system (figure 1) already reported the complex behavior of the dilation, being possible to separate it into two distinct parts (figure 2): one smooth, rather linear dilation (2d), and an intermittent multi-scale discontinuities in both directions (2b): dilation (called jumps) and contraction (called drops), with similar statistical distributions, (2c). In this work we include the analysis of the granular struc-

\footnotetext{
*e-mail: victor.levy-dit-vehel@univ-lyon1.fr

**e-mail: osvanny.ramos@univ-lyon1.fr

A video is available at https://doi.org/10.48448/mzkm-8r98
}

ture, which is able to corroborate the reported behavior, as well as proposing an explanation to the phenomenon, by considering an optimal angle in the direction of the force chains. A more detailed analysis of the correlation between dilation and avalanche size shows a continuous increase of the global dilation rate with the size of upcoming events.

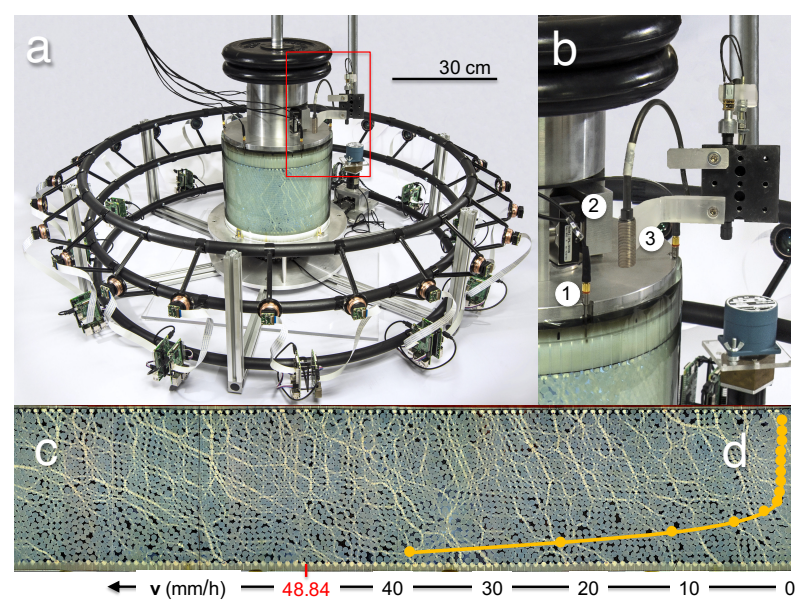

Figure 1. Experimental system. (a) Cylindrical cell containing photoelastic disks. 24 cameras placed in a ring takes images of the granular structure. (b) Close-up showing (1) acoustic sensor, (2) force sensor, and (3) capacitive sensor measuring vertical displacements of the upper plate. (c). Whole granular structure. Notice the force chains with different angles with respect to the vertical direction. (d) Velocity profile showing a clear shear band. The shear speed of the ring driving the system is highlighted. 

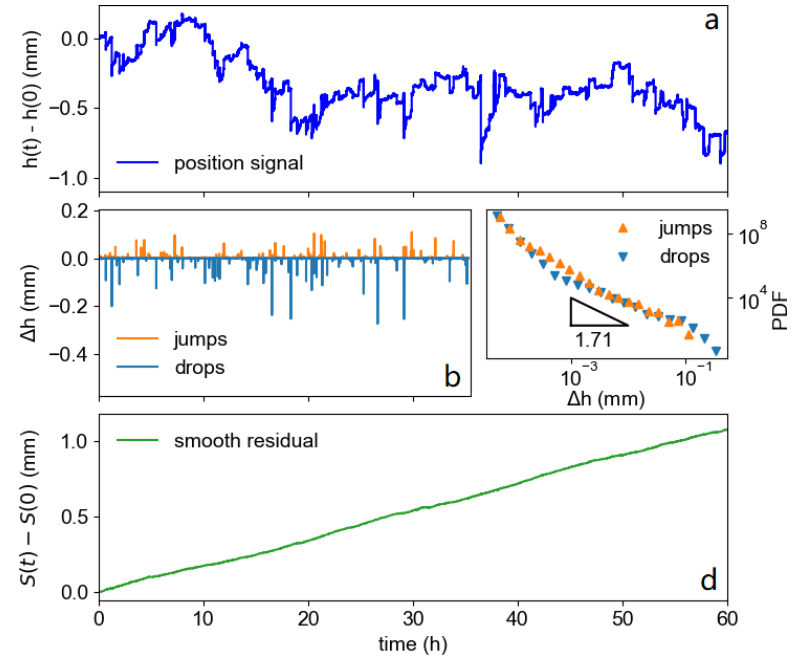

Figure 2. (a) The position signal $h(t)$ can be split into (d) a smooth dilation $S(t)$ and (b) sudden discontinuities (jumps or drops), denoted as $\Delta h$. (c) Discontinuities distribute following a power-law-like distribution over three orders of magnitude. The time $\mathrm{x}$-axis is shared for all plots except (c).

\section{Experimental system}

We study a bidisperse mixture of disks confined in one single layer between two concentric fixed acrylic cylinders (figure 1). The disks have been 3D-printed in Durus White 430 material -which is photoelastic- using an $\mathrm{Ob}$ jet30 printer. They have $4 \mathrm{~mm}$ thickness and diameters equal to $6.4 \mathrm{~mm}$ or $7 \mathrm{~mm}$ (in equal proportions). The granular layer is bounded by two rough circular rings where half-grains have been also 3D-printed. A dead load of $20 \mathrm{~kg}$ is placed over the top ring compressing the granular pile. The top ring is free to move vertically but not to rotate, while the bottom one is slowly rotated with a period of 18.33 hours, quasi-statically shearing the granular pile with a linear velocity of $48.84 \mathrm{~mm} /$ hour. The elastic energy slowly stored due to the shear is liberated by sudden events of all sizes that follows the laws of seismicity [7]. Thanks to a lever and a force sensor, we measure the torque $\Gamma(t)$ applied by the granular pile on the top ring. The vertical displacement $h(t)$ of the top plate, directly measuring the dilation of the granular medium, is monitored thanks to a capacitive sensor. The strict verticality of the movement has been verified with a second sensor and corroborated with image analysis. Sudden variations in $h(t)$ are denoted as $\Delta h$. if $\Delta h>0$ the medium dilates, if $\Delta h<0$ the medium contracts. Both measures are done at a rate of 100,000 samples per second. The system is left to evolve for 64 hours, generating more than 80,000 torque drops. The energy of torque drops are computed following the procedures detailed in [7]. Their distribution follows the same power law as in [7]: $P\left(E_{M}\right) \sim E_{M}^{-\beta}$, with $\beta=1.71$. In addition, 24 cameras take images of the granular structure at $4 \mathrm{~s}$ time intervals. They are limited to 22 hours.

\section{Jumps in dilation and force chains}

In our previous report we have shown that the distribution of jumps is similar to a power law for both dilation

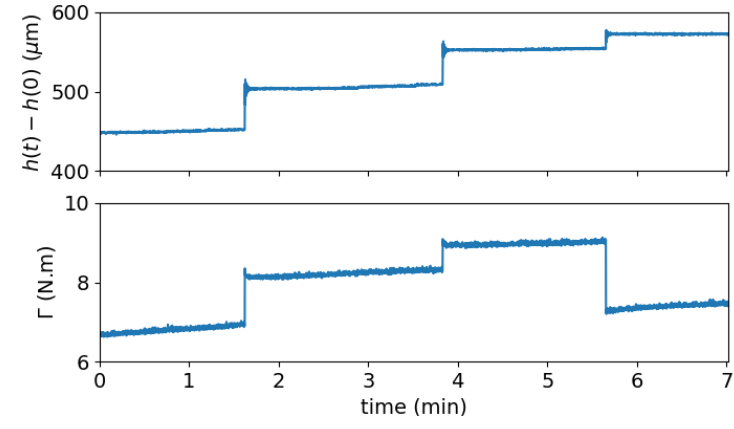

Figure 3. Sudden variations in $h$ are simultaneous with sudden variations in resisting torque $\Gamma$.

and contraction events (see figure 2c). Dilation jumps are more numerous, but contraction ones are bigger, dominating the dynamics and globally compensating the smooth increase of dilation [12]. The analysis of the granular structure, particularly the simple inspection of force chains [13-15], brings plausible explanations to the dynamics of jumps in dilation. Jumps are caused by the same rearrangement of grains as mechanical events are, and occur at the same time, as seen in figure 3. Considering that force is measured radially (as a resisting torque $\Gamma(t)$ ) and the $\Delta h$ events are related to vertical force (under constant pressure and free volume condition), it seems reasonable to assume that torque and vertical forces are simply orthogonal projections of the same force being applied at a given angle by the force chains (see figure 1c). When a force chain breaks, it is intuitive to think that the radial force will decrease and the now less supported top plate will drop $(\Delta h<0)$. However, figure 3 shows counterexamples to this intuitive view: From the three observable jumps, the first two correspond to sudden increases both in torque and $h(t)$, while the last jumps corresponds to a torque drop but with an associated increase of $h(t)$.

To investigate the relationship between these two measurements: $\Delta \Gamma$ and $\Delta h$, we need a procedure to identify which torque discontinuity $\Delta \Gamma$ matches which $\Delta h$. The inter-event time between either kind of measurement is very rarely below $100 \mathrm{~ms}$ while the time precision on event detection is in the range of $0.1 \mathrm{~ms}$. We have used a criterion of $\Delta t \leqslant 1 \mathrm{~ms}$ to consider that both measurements correspond to the same reorganisation event.

Matched events can then be normalized and compared in log-scale. The resulting scatter plot is presented in figure 4, where re-scaled $\Delta h$ are plotted against re-scaled mechanical energy changes (proportional to $\Delta \Gamma^{2}$ [7]). The scaling factor is, for each series, its detection threshold. In $\log$-scales, it ensures the range of values starts from 0 . The released energy associated to a torque drop is considered positive. Therefore a sudden increase of measured torque implies a "negative energy release".

Intuitively, most points should be in the bottom-right area of the graph (zone D), corresponding to release of mechanical energy and sudden compaction. However, a surprising feature emerges from the data. First, there are more "negative" energy release events than "positive" ones ( $46 \%$ and $54 \%$ respectively). Of course, energy is not sud- 


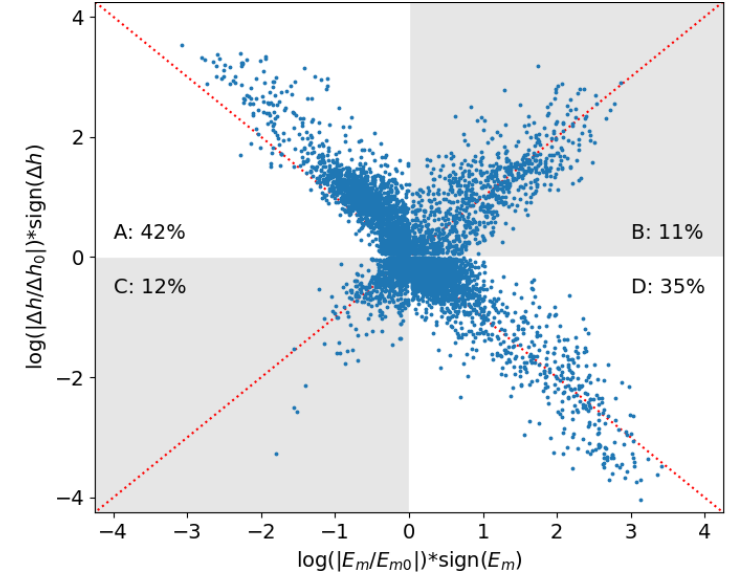

Figure 4. Relation between signed and normalized $\Delta h$ values and released mechanical energy, for matched events. A-D: four different behaviors with percentage of events in each classification.

denly created or injected in the system, as its only source is the motor slowly driving the shear: energy is injected at a low and near constant rate. While the applied stress at the bottom cannot increase abruptly, the way it is transmitted to the sensor may change.

Let us consider two extreme cases. First, a very strong and vertical force chain: it would not apply any force on the radially-oriented sensor. The same goes for a hypothetical perfectly flat chain, as it would not reach the sensor at all. In order to maximize their stability, force chains reorganize around a local optimal angle, placing themselves parallelly to the total stress. We will use this reasoning of a single one-chain description, where one chain (called "active") holding most of the stress is broken and replaced by a new one, as a main ingredient to explain all the four different behaviors presented in the figure 4:

A ( $\Gamma \uparrow, h \uparrow, 42 \%$ of events): negative energy release and dilation of the medium. Replacement of the active force chain with an equivalent one. This is actually the most common case, particularly for lower energy events. The second ingredient to explain the dynamics corresponds to the inertia of the structural reorganization: the kinetic energy developed by the system during the reorganization time, generates an extra-pressure in the new force chain, reading as an increase of the force in both lateral (torque) and vertical (dilation) directions with respect to the replaced one.

$\mathrm{B}$ ( $\Gamma \downarrow, h \uparrow, 11 \%$ of events): positive energy release and dilation of the medium. Replacement of the active force chain with a more vertical one. The horizontal projection of the force is smaller with respect to the replaced one. This reads as a reduction of the global torque and, thanks to the inertia, an increase in the vertical force, which provokes a dilation of the medium.

$\mathrm{C}(\Gamma \uparrow, h \downarrow, 12 \%$ of events): negative energy release and contraction of the medium. Replacement of the active force chain with a more horizontal one. It should increase the global torque thanks to a better projection; as well as bringing a contraction. However, the probability of finding such a percolating chain for high-energy events is rather low, because of its instability.

$\mathrm{D}(\Gamma \downarrow \downarrow, h \downarrow, 35 \%$ of events): positive energy release and contraction of the medium (intuitive case of a collapse). Replacement of the active force chain with a weaker one. The more energetic releases fall into this classification. Notice that from the 33 more energetic events, 31 events $(94 \%)$ fall in the D-zone and only $2(6 \%)$ in the A-zone.

This rich behavior of our granular fault is a direct consequence of the large number of force chains percolating our two-dimensional system (figure 1) holding both normal and shear stresses. The sudden and intermittent events that characterize the dynamics take place when an active force chain is replaced, in most cases, by a similar one (case A); or a weaker one (case D) particularly for the very stable configurations, leading to high energy events. The replacement for a more vertical chain (associated to the case B) seems a plausible explanation of the counterintuitive case presented in the last event of figure 3 . The replacement for a more horizontal chain (case C) is less stable and therefore uncommon for high-energy events.

Besides the four different scenarios, it is clear that any change in volume is correlated with the intensity of the rearrangement event. This correlation is indeed observed in figure 4, as events are clustered around either the identity diagonal or the counter-diagonal.

\section{Precursory behavior}

The results of figure 4 (D-zone) show that high-energy events are strongly correlated with a sudden contraction of the system. This is an indication that a volume increase, i.e., a dilation, is expected preceding those events.

To corroborate this assumption we have analyzed the evolution of the structure of the system around the 33 largest mechanical events. A clear shear band (figure 1d) divides the structure into a very mobile zone, corresponding to a layer of about 10 grain diameters adjacent to the

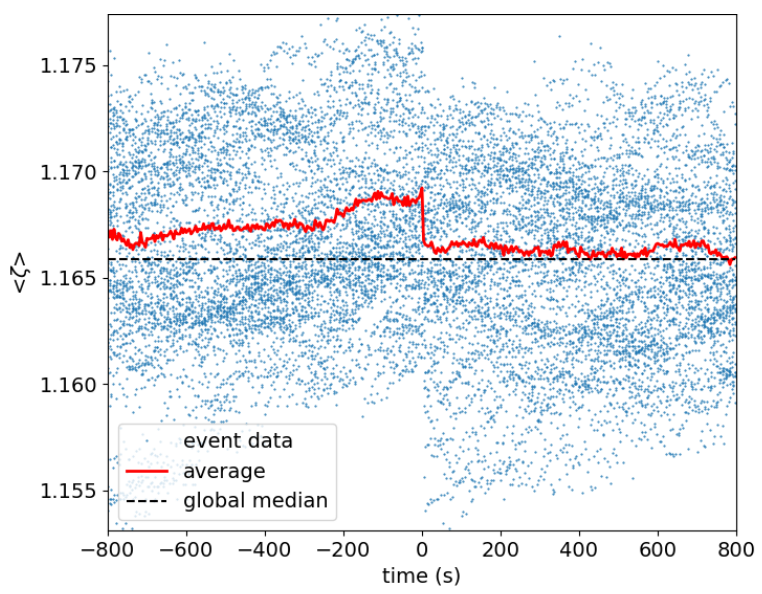

Figure 5. Each dot corresponds to the $\zeta$ parameter (inversely correlated with local density) averaged within the shear band on one picture at a given time $t$ from the 33 largest events. Solid curve: averaged over the 33 images found at a given time $t$. 


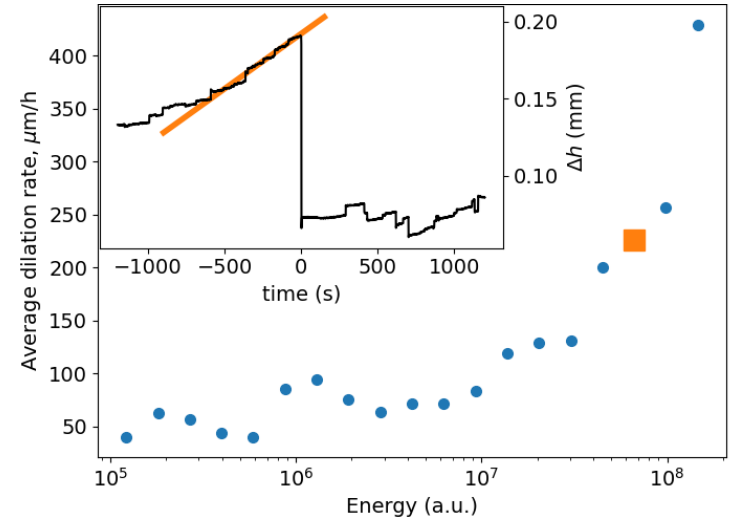

Figure 6. Dilation rate plotted against binned event energy. As events get smaller, the slope continuously decrease to a plateau. The dilation rate is computed by a linear regression on 300 s, as shown in the insert, and this particular point is highlighted as an orange square on the main curve.

moving ring at the bottom of the system; and a zone that is almost static in the top part of the pile [7, 16, 17]. We have focused our analysis on the shear band.

The local structure around single grains can be probed by a shape factor $\zeta=P^{2} / 4 \pi A$, defined as the ratio between the perimeter $P$ and the area $A$, of the cell of each grain in a Voronoi tessellation [3]. As $\zeta$ is normalized to a circle, a regular hexagon, which is the minimum possible value in our structure, has $\zeta=1.103$, a square has $\zeta=1.273$ and a particle neighboring a hole in a hexagonal lattice has $\zeta=1.286$. This $\zeta\left(x, y, t-t_{N}, N\right)$ is computed for every disks at position $(x, y)$ within the shear band of every image and at a given time $t$ with respect to the time of a large event $t_{N}$, where $\mathrm{N}$ runs from 1 to 33 containing the 33 largest events. $\zeta$ is first averaged under every image, corresponding to the dots on figure 5 . The $\zeta\left(t-t_{N}, N\right)$ values of all 33 images found in the same time windows of $4 \mathrm{~s}$ width are averaged, resulting in $\langle\zeta\rangle(t)$, corresponding to the solid curve on figure 5. Both averages are shown $800 \mathrm{~s}$ both before and after the events happening, with a horizontal black line to show the global median. In the dots, and much more clearly on the average, a dilation emerges from roughly 10 minutes prior to the events. For comparison, the average time between selected events is around 40 minutes (22h of images available).

The behavior described so far emerges from data related to "sufficiently large" events. This implies threshold choices, which may have an influence on the results. To explore the robustness across size, we have measured the average dilation prior to events binned by size, a measure first done for the largest event only in [12]. As events are binned, the content of each bin is independent: the largest event appears in the highest bin and not in any bin below. Figure 6 shows that the average dilation rate seems monotonically dependent on the binned energy. This relation lends weight to the precursory nature of dilation, as event energy and average expansion appears directly related.

\section{Conclusion}

We have analyzed the dilation of a compressed granular fault under slow shear showing the existence of four different behavior associating volume contraction and dilation with positive and negative jumps in the resisting torque. We have explained those behaviors by analyzing the replacement of an active force chain with a new one of a given strength and orientation, and considering the existence of a local optimal angle in the direction of force chains, which place themselves parallelly to the total applied stress. We have also shown that the average rate of increase of global dilation varies monotonically with the size of the energy release event, making dilation a plausible candidate to predict catastrophic events in such earthquake-like systems.

We acknowledge financial support for the AAP-iLM-2020 and the LIA D-FFRACT.

\section{References}

[1] P.A. Johnson, H. Savage, M. Knuth, J. Gomberg, C. Marone, Nature 451, 57 (2008)

[2] K.E. Daniels, N.W. Hayman, J. Geophys. Res. Solid Earth 113, 2156 (2008)

[3] O. Ramos, E. Altshuler, K.J. Måløy, Phys. Rev. Lett. 102, 078701 (2009)

[4] J. Rivière, Z. Lv, P. Johnson, C. Marone, Earth Planet. Sci. Lett. 482, 407 (2018)

[5] A. Petri, Experimental statistics and stochastic modeling of stick-slip dynamics in a sheared granular fault (Società Italiana di Fisica, 2018), Vol. 202, p. 113

[6] A. Abed Zadeh, J. Barés, J.E.S. Socolar, R.P. Behringer, Phys. Rev. E 99, 052902 (2019)

[7] S. Lherminier, R. Planet, V. Levy dit Vehel, G. Simon, L. Vanel, K.J. Måløy, O. Ramos, Phys. Rev. Lett. 122, 218501 (2019)

[8] D. Houdoux, A. Amon, D. Marsan, J. Weiss, J. Crassous (2020), arXiv:2007.02867

[9] P. Bak, C. Tang, K. Wiesenfeld, Phys. Rev. Lett. 59, 381 (1987)

[10] C.H. Scholz, Geology 15, 493 (1987)

[11] O. Reynolds, Lond.Edinb.Dubl.Phil.Mag. 20, 469 (1885)

[12] V. Levy dit Vehel, F. Dubourg, L. Vanel, K.J. Måløy, O. Ramos, Evolution of the distance between plates in an experimental granular fault. Implications for earthquake forecast (Non-Linéaire Publications, 2018), pp. 43-48

[13] T.S. Majmudar, R.P. Behringer, Nature 435, 1079 (2005)

[14] K.E. Daniels, J.E. Kollmer, J.G. Puckett, Rev. Sci. Instrum. 88, 051808 (2017)

[15] S. Lherminier, R. Planet, G. Simon, L. Vanel, O. Ramos, Phys. Rev. Lett. 113, 098001 (2014)

[16] O. Kuwano, R. Ando, T. Hatano, AIP Conf. Proc. 1542, 32 (2013)

[17] D.L. Henann, K. Kamrin, PNAS 110, 6730 (2013) 